\title{
Information Retention During Competitive Interactions: Siblings Need to Constantly Repeat Vocal Displays
}

\author{
Amélie N. Dreiss • Charlène A. Ruppli • \\ Sévrine Antille • Alexandre Roulin
}

Received: 26 May 2014/ Accepted: 13 November 2014/Published online: 28 November 2014

(C) Springer Science+Business Media New York 2014

\begin{abstract}
Memory is essential to adjust behaviour according to past experience. In societies where animals interact on numerous occasions, memory of previous social interactions may help optimise investment in competition. How long information about the resource holding potential and motivation to compete of conspecifics is retained depends on how fast the value of this information fades, but also on the cost and benefit of retaining information. Information retention has never been investigated in the context of interactions prevailing within the family and more specifically sibling competition. In the absence of parents, barn owl (Tyto alba) nestlings vocally compete for priority of access to the next indivisible food item brought by a parent. The finding that owlets eavesdrop on vocal interactions between siblings to adjust investment in vocalization once competing with them suggests that they memorize siblings' vocal interactions. Playback experiments showed that owlets take into account the past siblings' vocal performance that signals hunger for at least $15 \mathrm{~min}$, but only if the performance was witnessed during a sufficiently long period of time (30 min). Moreover, using natural vocal exchanges in another set of individuals, we showed that sibling signalling was no more taken into account after a few minutes. This suggests that young barn owls need to continuously display their motivation to trigger siblings' withdrawal from the current competition. Repeating a vocal display may ensure its honesty. Studying the extent to which individuals retain past information is important to understand how individuals adjust their competitive investment over resources.
\end{abstract}

A. N. Dreiss $(\bowtie) \cdot$ C. A. Ruppli · S. Antille · A. Roulin

Department of Ecology and Evolution, University of Lausanne,

Building Biophore, 1015 Lausanne, Switzerland

e-mail: Amelie.Dreiss@unil.ch
Keywords Eavesdrop $\cdot$ Memory $\cdot$ Negotiation $\cdot$ Sibling competition - Communication $\cdot$ Signalling $\cdot$ Parentoffspring conflict

\section{Introduction}

Information retention can be of great adaptive value if it helps individuals to remember the location of the best food patches and nest sites (Martin 1995; Clayton and Dickinson 1998) or to adjust behaviour to predation risk (Brown et al. 2006). Relying on memory is beneficial as long as the derived benefits of taking the correct behavioural decision based on past interactions outweigh the associated cognitive costs of retaining information (Dukas 1999; Mery and Kawecki 2005). Memory can though become useless when the retention of past information loses relevance, because external factors (such as food supply) or internal factors (such as individual condition) have modified the competitive environment (Dunlap et al. 2009). The way animals memorize and forget information (Killeen 1981; McNamara and Houston 1987) has important implications on the evolution of cooperation (Moreira et al. 2013) and on decision-making at different life stages, for mate choice (Castellano et al. 2012), foraging (Greggers and Menzel 1993; Devenport et al. 1997) or agonistic interactions (Dedeo et al. 2010). However, to the best of our knowledge, the duration of memory retention has never been considered as an important component of interactions prevailing between members of the same family. For instance, if an individual memorizes the previously witnessed signals of need displayed by siblings, this information about siblings' hunger level and motivation to compete for parental food resources should influence the way this individual will adjust the level of competitive investment once parents are back with food. 
In a socially complex environment, memory of previous social interactions in terms of recognizing competitors and their competitive level is found in various species including fish, birds, mammals and crustaceans (Mennill and Ratcliffe 2004; Grosenick et al. 2007; Aquiloni and Gherardi 2010), but the duration of social information retention has rarely been tested experimentally. In social groups, animals can present long-term memory of the identity of group members based on face characteristics for over 2 years in sheep Ovis aries (Kendrick et al. 2001) or on song characteristics during 8 months in hooded warblers Wilsonia citrina (Godard 1991). Ravens Corvus corax have also recently been shown to differentiate individuals with which they had shared a valuable affiliate relationship up to 3 years ago (Boeckle and Bugnyar 2012). In the hermit crab (Pagurus longicarpus) only one competitive interaction is sufficient for individuals to identify an opponent, evaluate the probability of success when competing again against this opponent and to form a stable dominance hierarchy (Gherardi and Atema 2005). Thus, based on only a single interaction crabs memorize their relative position in the social hierarchy during several days without having to interact again with competitors (Gherardi and Atema 2005). In territorial birdsongs, memory of social relationships between males can last at least one night, but has not been tested for longer periods of time (Erne and Amrhein 2008; Schmidt et al. 2007).

Social information can hence be retained for very long periods of time, as long as it concerns stable traits such as position in social hierarchy. In contrast, it is still unknown for how long individuals memorize information about traits that are more variable in time (e.g. hunger level and motivation to compete). In young altricial animals, siblings compete over the share of parental resources on multiple occasions during their development (Godfray 1995). Young animals might not only remember the outcome of their own social interactions with given conspecifics, but also the outcome of previous interactions they could observe between two or several conspecifics (i.e. social eavesdropping) in order to adjust their own behaviour (Dreiss et al. 2013a). Memory of siblings' previous interactions may be beneficial if it allows young to assess more accurately the motivation of their siblings to compete, and hence to optimally adjust their own competitive effort. However, not taking into account past interaction may oblige competitor siblings to constantly repeat their signal of motivation to reinforce the honesty of sibling communication. Moreover, memorizing sibling interactions may not be an easy task due to the dynamic nature of competitive interactions taking place between young animals competing over resources that parents provide at regular time points. For instance, in passerines, parents can bring food to their nest thousands of times per day and the numerous offspring compete at each parental feeding visit. Because the hunger level of each nestling can change rapidly, retention of siblings' motivation to compete should not be longer than the time span between two siblings' meals.

Here, we investigate information retention of social interactions taking place between young siblings in the barn owl Tyto alba. In this nocturnal species, nestlings exchange hissing calls thousands of times per night when waiting for the next parental feeding visits (Roulin 2002a). The outcome of these sib-sib vocal interactions, so-called "sibling negotiation", influences which individual will have priority access to the next delivered indivisible food item (Johnstone and Roulin 2003; Roulin et al. 2000; Dreiss et al. 2010b). Typically, hungry individuals vocalize intensely in the absence of parents to deter their siblings from competing at the parents' arrival, producing higher call rate and longer calls and responding faster to siblings' calls (Dreiss et al. 2013a; Roulin et al. 2009). As a consequence, at a given level of hunger, an individual will call at a lower level when its siblings are highly motivated to compete for food than when its siblings are sated, since the probability to obtain the next delivered food item is lower in front of hungry than food-satiated siblings (Roulin et al. 2000). A recent playback experiment showed that nestling birds eavesdrop on conspecifics' behavioural interactions to gain information about the state of surrounding competitors without having to directly participate in these interactions (Dreiss et al. 2013a), similarly to interactions taking place between adult animals (McGregor 1993). After having listened to two siblings that interacted vocally, singleton owlets were allowed to interact with only one of these two individuals. Singletons vocalized less rapidly when interacting with the individual that previously displayed a higher motivation to compete compared to when interacting with the other individual, formerly less motivated (Dreiss et al. 2013a). This study demonstrates the ability not only to recognize the identity of individuals taking part in a social contest, but also to memorize during at least $1 \mathrm{~min}$ the outcome of these vocal interactions in order to use this information once interacting with only one of the participants. This recognition is facilitated by the presence of vocal signatures (Dreiss et al. 2014). Barn owls have been shown to have the cognitive ability to perceive information on social context, such as motivation of nest mates (Ruppli et al. 2013a) and amount of competing siblings (Ruppli et al. 2013b), but the duration of social information retention is unknown. Because parent feeding visits occur at unpredictable time points (Roulin and Bersier 2007), siblings vocal exchange between two feedings can last up to several hours (Roulin 2002b). We hence investigated how long information on sibling motivation influence young competitive behaviour. 
To answer this question, we first investigated for how long a signal experienced at a given time point influences opponent's behaviour. Although the duration of calls emitted at different time points is repeatable within individuals (Dreiss et al. 2014), it fluctuates over time independently of hunger, since siblings constantly adjust their call duration to one another (Roulin et al. 2009). As a consequence, the call durations of a vocalizing pair of siblings fluctuates synchronously (Roulin et al. 2009). Using correlative data, we therefore analysed for how long the duration of siblings' calls was taken into account by focal nestlings to adjust the duration of their own calls.

Second, we used the barn owl's ability to eavesdrop on vocal interactions (Dreiss et al. 2013a) to test experimentally (1) how long nestlings retain an eavesdropped interaction between two competitors and use this information to adjust vocal behaviour, i.e. the duration of the "memory window", and (2) whether repetition of the eavesdropped vocal interaction (i.e. the number of "training") consolidates young propensity to use the eavesdropped information. The key issue is after how long without any vocal information, nestlings stop responding differently to two competitors that previously displayed different motivation levels. Under the hypothesis that the memory decreases with the time lapse since the social information was witnessed, the response to the two competitors should only differ after shorter memory windows. Under the hypothesis that memory increases with the repetition of the social information, the response to the two competitors should only differ after more trainings. We indeed expected that nestlings would better remember the vocal interaction when they heard it repeatedly. Under the hypothesis that nestlings memorize vocal interactions that took place between siblings, singleton nestlings should show lower motivation to vocalize (i.e. produce fewer and shorter calls but also vocalize with longer time latency after playback calls) when hearing an individual that was previously witnessed as "motivated" (i.e. high call rate) compared to "demotivated" (Dreiss et al. 2013a; Roulin et al. 2009).

\section{Materials and Methods}

\section{General Procedure}

We performed the study in western Switzerland $\left(46^{\circ} 4^{\prime} \mathrm{N}\right.$, $6^{\circ} 5^{\prime} \mathrm{E}$ ) on a population of wild barn owls breeding in nest boxes. In this species, incubation starts as soon as the first egg has been laid and since eggs are laid every 2.5 days, the two to nine siblings can differ strongly in age. Nestling age was estimated shortly after hatching by measuring the length of the left flattened wing from the birds' wrist to the tip of the longest primary (Roulin 2004). To record young vocal exchange and their response to an owlet playback, we temporarily brought young in an experimental nest box similar to the one where they were reared in naturally $\left(62 \times 56 \times 37 \mathrm{~cm}^{3}\right)$, but separated into two equal parts by a thin wooden wall pierced with holes. One owlet was placed at one side of a box, while the other side contained either a sibling or a loudspeaker to broadcast pre-recorded calls (near05 experience, ESI Audiotechnik GmbH, Leonberg, Germany). During the course of the experiments, owlets did not receive any food item, simulating a situation where nestlings wait for parental feeding. The relative motivation level of each individual should thus have remained the same during these $4 \mathrm{~h}$. We recorded each individual with a microphone (MC930, Beyerdynamic GmbH \& Co KG, Heilbronn, Germany) oriented towards it and fixed on the inside roof of the box. We tested owlets on average 22 days before fledging, which takes place at ca. 55 days. At the tested age owlets are already thermoindependent and their parents are naturally sleeping outside their nest box.

\section{Natural Vocal Exchange Between Siblings}

In 2008, we analysed the correlation of call durations between pairs of siblings in 98 nestlings issued from 35 broods (51 males, 45 females and two of unknown sex; aged 25-45 days, mean \pm SD: $35 \pm 5$ days) during a vocal exchange. Pairs of siblings were recorded during $4 \mathrm{~h}$ from 19:30 to 23:30, two consecutive nights, alternatively in food-satiated and food-deprived states (see Ruppli et al. 2013a for further details on procedure). We analysed the temporal auto-correlation of continuous vocal exchanges between food-deprived pairs. We used hungry nestlings, because they vocalized more (Ruppli et al. 2013a) and we could analyse long series with continuous call production.

\section{Playback Experiment}

In 2011, we brought 115 owlets to the laboratory, including 52 males, 58 females and 5 nestlings of unidentified sex, from 32 broods (aged 20-42 days, mean \pm s.e.: $33.4 \pm$ $0.4)$. At their arrival to the laboratory at around 12:00 and on the next morning at 8:00 nestlings received ca. $50 \mathrm{~g}$ of laboratory mice, which is slightly below their daily food requirement of ca. $67 \mathrm{~g}$ (Durant and Handrich 1998). The playback experiment was carried out on the second and last night of captivity starting at 21:30.

We first constructed an artificial vocal exchange between two individuals, so-called "competitors", each presenting a different level of motivation to negotiate. This training playback lasted 10 min and comprised 16 calls per minute, 12 calls from one "motivated" individual and 4 calls from the second unrelated "demotivated" individual 
(Fig. 1). We generated this asymmetry in call rate to mimic a situation where the singleton nestling hears two competitors showing a clear difference in the motivation to compete. This call rate is in the natural range of call rates observed in dyadic interaction (Ruppli et al. 2013a). In nature, hungry nestlings call on average nine times more often than their satiated sibling (unpublished data) and hungry nestlings produce longer calls than satiated ones (Roulin et al. 2009; Ruppli et al. 2013a). Our experiment is therefore conservative and a larger difference in the intensity of the competitors' motivation (in terms of repetition and magnitude) during the training playback, as well as more repetitions of this playback, may elicit a longer memory. Calls were inserted in a random order along the soundtrack and at random time onset (with a minimum $1 \mathrm{~s}$ pause between two successive calls), so that each nestling heard three different soundtracks and no nestlings heard the same soundtrack.

After having broadcast the training playback to the singleton nestlings, we examined their vocal reaction when facing again the calls of only one of the two broadcast competitors during a testing playback (Fig. 1). To test nestlings' memory, we inserted a silent "memory window" of 1,15 or $30 \mathrm{~min}$ between the end of the training playback and the beginning of the testing playback. We chose these memory windows because parents visit their nest on average every half an hour during the night in natural conditions (median of 17 broods of 2.4 nestlings, median $=27 \mathrm{~min}$, range $(1 \mathrm{~min}-5 \mathrm{~h})$, unpublished data). As nestlings' hunger levels should not importantly vary between two feeding events, it may be biologically relevant to memorise the hunger level of siblings during this time lapse. In this testing playback, we sequentially broadcast the "motivated" and the "demotivated" competitors alone in a random order: during 5 min the calls of a first competitor followed by a period of $30 \mathrm{~s}$ of silence and then the calls of the second competitor during 5 min (Fig. 1). Playback calls of formerly "motivated" and "demotivated" individuals were broadcast at a rate of 5 calls per minute.

Each singleton heard three times in a row a sequence containing a training playback, a silent memory window and a testing playback with a period of $10 \mathrm{~min}$ of silence after the testing playback (Fig. 1). The duration of silent memory window varied between the three repetitions from 1,15 to $30 \mathrm{~min}$, the order being randomized for each singleton nestling. For a given singleton nestling, in each of the three training playbacks, we used the same individual to play the role of a "motivated" competitor and the same individual to play the role of a "demotivated" competitor. However, the playback calls used to build the training playbacks were reallocated at random timing and random order in each of the three training playbacks so that the three sequences of calls were different. For a given nestling, the order at which the "motivated" competitor was broadcast during the testing playback (first or second) was the same the three times.

\section{Playback Soundtracks}

To build the playback soundtracks, we used calls we had recorded in 2008, of food-deprived nestlings (Roulin et al. 2009; Ruppli et al. 2013a). We selected calls from five individuals of similar age (33-35 days old; three males and two females) issued from five different broods. For each of these five unrelated individuals, we isolated 10 calls of about $0.6 \mathrm{~s}$ (mean \pm s.e.: $0.597 \pm 0.002$ ), which corresponds to the first quartile of call duration computed from 54 experimentally food-satiated pairs of nestlings recorded in 2008 (0.61 s, based on 96,666 recorded calls) (Ruppli et al. 2013a). We choose to broadcast calls of relatively short durations, because at the beginning of the night when

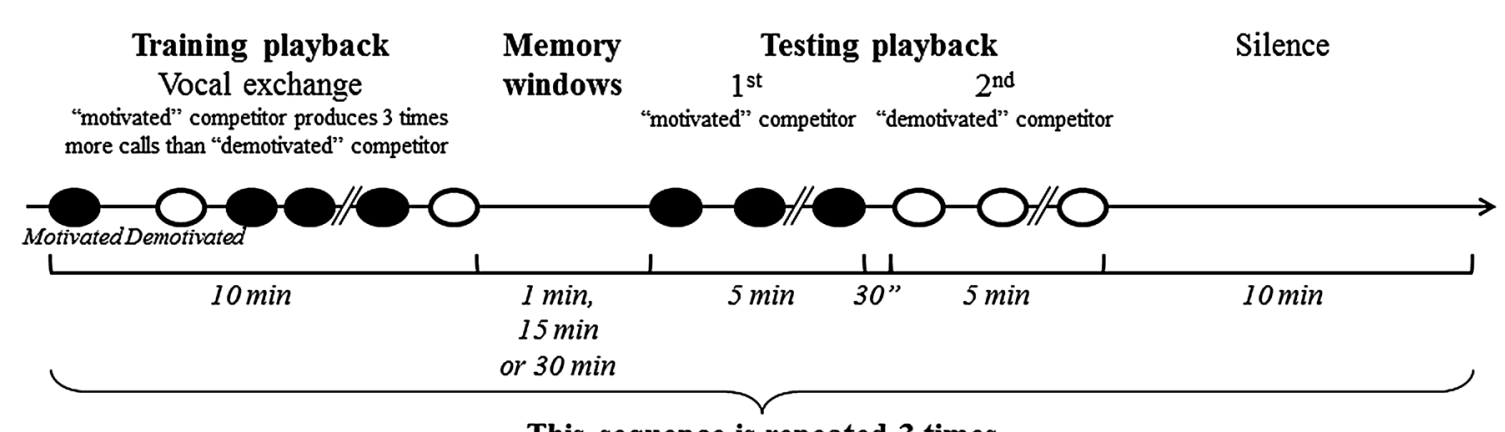

This sequence is repeated 3 times

Fig. 1 Design of the experimental playback sequence used to test the ability of barn owl nestlings to memorize vocal interactions taking place between two siblings. The sequence was broadcast three times in a row to each singleton nestling with each time a different silent memory window of 1,15 or $30 \mathrm{~min}$, the order of each window being randomized across nestlings. The order at which the "motivated" and "demotivated" individual was broadcast in the testing playback was randomised across singleton nestlings (but for each nestling the order was the same for the three testing playbacks) 
the experiment started, nestlings were relatively satiated and would probably not respond to highly motivated individuals producing long calls. We normalised the maximum loudness of all playback calls at $-3 \mathrm{~dB}$ using the Audacity software (http://www.audacity.sourceforge. net); this manipulation did not affect the other acoustic features of calls (such as call duration or frequency distribution). Calls of the motivated and demotivated nestlings during the training and the testing playbacks were hence of same intensity. The five playback individuals were equally used to play the role of "motivated" and "demotivated" competitor $\left(\chi^{2}=1.88, d f=4, P=0.77\right)$.

\section{Acoustic Analyses}

We used Matlab v.7.7 (MathWorks, Natick, MA, U.S.A.) to assign the recorded calls to the broadcast individuals and to live nestlings (see Ruppli et al. 2013a for further details on procedure) and (see script in Dreiss et al. 2013a). For playback experiment, we measured the duration and the number of calls produced by the singleton nestlings during the $10 \mathrm{~min}$ of the three testing playbacks. We also determined the timing when each call had been emitted in order to measure the latency of response by the singleton nestlings after the broadcast calls, i.e. the duration of the pause between every broadcast calls that were followed by a call of the live singleton nestlings. This duration, referred to as "calling latency", was defined as the amount of time between the beginning of a broadcast call and the beginning of the singleton nestling's call.

\section{Statistical Analyses}

\section{Natural Vocal Exchanges}

For each focal individual, we analysed the relationship between its call duration and the duration of the preceding calls emitted by its sibling. To simplify the analyses, we averaged the duration of the calls produced each minute $t$. In order to control statistically for the temporal autocorrelation of call durations, we extracted the residuals from the regression of mean duration of sibling's calls at minute $t-1$ against mean duration of sibling's calls at minute $t$. This value allows us to estimate the residual effect of minute $t-1$, given the temporal correlation between sibling call duration at $t$ and $t-1$. We repeated this procedure for each time step (i.e. minute $t-2$ against minute $t-1$ and $t$, and so on until minute $t-10$ ). Sibling's call duration at minute $t$ and the sibling's call duration residual values at minutes $t-\mathrm{x}$ were by construction not correlated (Pearson correlations, all $P$ values $>0.10$ ). The entire procedure was repeated for the calls produced by focal nestlings. In this way, we had repeated measures for each pair of individuals that could be analysed without the problem of temporal autocorrelations.

We used linear mixed models with call duration of focal individual at minute $t$ as dependent variable and as covariates sibling call duration at minute $t$ and residuals of sibling call duration at minutes $t-1$ to $t-10$. In a second model, covariates were the residuals of focal individual call duration at minutes $t-1$ to $t-10$. The identity of each nestling nested in the pair of siblings was set as random factors and time of the night in minutes as covariate. The estimates of model effects give an indication on whether past call duration was taken into account.

\section{Playbacks}

To investigate whether singleton nestlings adjusted their vocal behaviour during the testing playback in relation to the role played by the broadcast competitors ("motivated" vs. "demotivated") during the training playback, we analysed three vocal parameters as dependent variables in separate mixed models (number of calls, mean call duration and mean calling latency). As independent factors, we fitted (1) the competitor motivation during training playback (i.e. "motivated" or "demotivated"), (2) the duration of the memory window after the previous training playback (i.e. 1, 15 or $30 \mathrm{~min}$ ), (3) the number of times singleton nestlings heard the training playback (1, 2 or 3 times), and (4) the order with which the calls of the "motivated" individual had been broadcast during the testing playback (i.e. before or after the "demotivated" individual). We predict that if nestlings take into account the information gathered during the training playback, they would respond differently to the two playback competitors during the testing playback, and the factor "competitor motivation" would be significant. If this discrimination depends upon the duration of the silence between the training and test playbacks, the interaction term "competitor motivation $\times$ duration of the memory window" would be significant. If it depends upon the number of training playbacks heard, the term "competitor motivation $\times$ number of training playback" would be significant.

Analyses were based on six average values per nestlings, as all nestlings were tested during three testing playbacks following three different levels of "Duration of memory window"; and each testing playback comprised the two levels of "Motivation of competitors". Because some nestlings did not call during some testing playbacks, sample size of call duration and calling latency is smaller than sample size of number of calls. We included the identity of the singleton nestlings nested in the brood from which they were issued as random factor. As we broadcast calls of five competitor nestlings to several singleton nestlings, we also controlled for the identity of the two 
broadcast competitor nestlings as random factor in all models. Call duration and calling latency of singleton nestlings were analysed with linear mixed models, while number of calls was analysed with generalized Poisson mixed model for zero-inflated over-dispersed count data (Joe and Zhu 2005). In all analyses, age and sex of the singleton nestlings did not affect the way they vocalized when hearing the testing playbacks and hence we did not include these variables in the models presented in the paper for the sake of clarity. Assumptions for the models (homoscedasticity and normal distributions of variables or residuals) were verified in each test.

51 nestlings (44\%) did not produce any call during the testing playbacks and were thereby not included in analyses. This is not surprising because the testing phase lasted only $10 \mathrm{~min}$ and nestlings were not very hungry, as they received food during the daylight hours and playback was performed at the beginning of the night. Depending on when the food was eaten (which was not standardized), some nestlings were hungrier than others. We predicted that singleton nestlings should call more in front of "demotivated" competitor than "motivated" competitor as predicted by the "sibling negotiation hypothesis" (Roulin et al. 2000); this prediction holds whatever the level of hunger of the singleton nestlings, as long as they are hungry enough to enter the contest. However, nestlings are predicted not to call at all in front of vocal competitors if not enough hungry as predicted by a game-theoretical model and as already shown in natural conditions (Johnstone and Roulin 2003; Roulin 2002a). These silent individuals did neither differ in age nor sex from the vocal individuals (age: ANOVA: $\mathrm{F}_{1,113}=0.16, P=0.67$; sex: $\chi^{2}=0.27, d f=1, P=0.60$ ). The probability that a singleton nestling was silent did not depend on which of the 5 playback individuals was used for the training playback ("motivated" competitor: $\chi^{2}=2.28, d f=4, P=0.69$; "demotivated" competitor: $\chi^{2}=2.05, d f=4, P=0.73$ ).

To control for the randomization of our protocol, we verified that among the 64 nestlings that vocalized, they neither differed in age nor sex according to the six different possible orders of silent memory windows (1/15/30, 1/30/ $15,15 / 1 / 30,15 / 30 / 1,30 / 1 / 15,30 / 15 / 1 \mathrm{~min}$; age: ANOVA: $\mathrm{F}_{5,58}=0.45, P=0.81$; sex: $\chi^{2}=6.54, d f=5, P=0.26$ ) and to the order at which the "motivated" and "demotivated" competitor nestlings were first broadcast (for a given singleton nestling, the same individual was broadcast first in all three testing playbacks, age: ANOVA: $\mathrm{F}_{5,62}=1.61, \quad P=0.21 ; \quad$ sex: $\quad \chi^{2}=0.07, \quad d f=1$, $P=0.80)$.

We used SAS v.9.1 (SAS Institute Inc., Cary, NC, USA) for all statistical analyses. Model selection was performed by backward elimination of the non-significant $(P<0.05)$ terms beginning with the highest order interaction terms.
Elimination of non-significant terms did not significantly modify the Akaike information criterion (AIC). Final models only contained significant effects and when a twoway interaction term was significant, the main effects involved in the interaction were retained in the model.

\section{Ethical Note}

In the laboratory, barn owl nestlings behave as in nature (Roulin et al. 2009) and are not physiologically stressed (Dreiss et al. 2010a). The stay at the laboratory did not affect nestlings' growth and survival, as there was no significant differences between siblings tested in the laboratory and siblings that remained in their natural nest (Wilcoxon tests: body mass at fledgling: $\mathrm{Z}=0.69$, $P=0.49$; survival: $\mathrm{Z}=1.16, P=0.25$ ). See (Dreiss et al. $2013 b$ ) for further details on transportation and feeding in the laboratory.

\section{Results}

\section{Natural Vocal Exchange Between Siblings}

The mean duration of calls produced by focal barn owl nestlings at minute 0 was positively correlated with the duration of calls produced by their sibling during the same minute and during the preceding $12 \mathrm{~min}$ (Fig. 2a). The focal nestling's call duration was positively correlated with residual value of sibling call duration (which controls for temporal auto-correlation) of the preceding $6 \mathrm{~min}$ (Fig. 2b; Table 1). This means that the call duration at minute 0 was positively correlated with sibling call duration at minute -6 , independently of the minutes -1 to -5 . As shown is Fig. $2 b$, the potential influence of sibling calls faded rapidly.

By comparison, within individuals, nestling call duration was highly positively correlated with duration of own calls emitted the preceding 20 min (Fig. 2a). The residual value of focal call duration was highly correlated with own call duration during the preceding $3 \mathrm{~min}$, but this correlation dropped after 3 min (Fig. 2b; Table 1).

\section{Playback Experiment}

Call duration of singleton nestlings during the testing playbacks was related to the interaction between competitor motivation during the training playback and the number of training playbacks they already heard (term "Motivation $\times$ Number" in Table 2b). Nestlings produced longer calls when interacting with the former "demotivated" than "motivated" competitor but only after having heard three times the testing playback (Fig. 3). The effect 


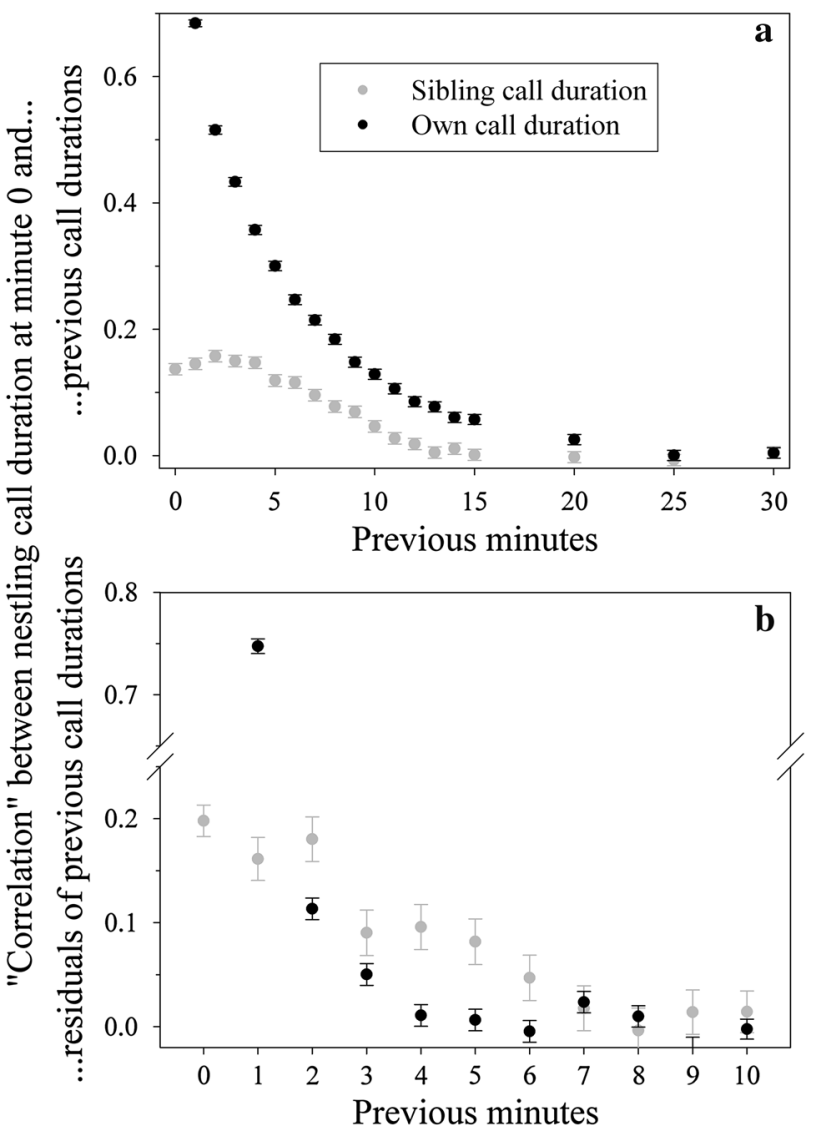

Fig. 2 Nestling call duration according to the duration of calls previously produced by the same individual or its sibling. Relationship between nestling call duration at minute 0 and a sibling call and own call durations the preceding minutes and $\mathbf{b}$ residuals of sibling call and own call durations the preceding minutes, which controls for temporal auto-correlation. Values are estimates $( \pm$ s.e.) of linear mixed models

of competitors' motivation was significant only after having heard the 3rd training playback (call duration: $-0.032 \pm 0.015, \mathrm{~F}_{1,32}=4.68, P=0.038$ ) but not after having heard the 2nd (call duration: $0.004 \pm 0.014$, $\mathrm{F}_{1,23}=0.11, P=0.75$ ) or 1st training playback (call duration: $-0.011 \pm 0.019, \mathrm{~F}_{1,23}=0.34, P=0.56$ ). Nestlings adjusted call duration independently of the duration of the last memory window (as terms "Motivation $x$ Duration $\times$ Number" and "Motivation $\times$ Duration" were not significant, Table 2a). This suggests that the duration of memory window within the experience had little effect on the discrimination of playback motivation. However, the only significant discrimination during the 3rd testing playback was found after $15 \mathrm{~min}$ of silence window (same models performed on the 3rd testing playback, when broadcast $1 \mathrm{~min}$ after training: estimate: $-0.01 \pm$ $0.02, \mathrm{~F}_{1,10}=0.17, P=0.6 ; 15$ min after: $-0.06 \pm 0.02$, $\mathrm{F}_{1,13}=0.78, \quad P=0.037 ; 30 \mathrm{~min}$ after: $-0.03 \pm 0.02$, $\left.\mathrm{F}_{1,7}=0.78, P=0.4\right)$.
Table 1 Relationship between nestling call duration at minute 0 and (a) sibling call duration the same minute and the residual of sibling call duration the 10 preceding minutes and (b) own call duration the preceding minute and the residual of own call duration the 2-10 preceding minutes, in two linear mixed models

\begin{tabular}{|c|c|c|c|c|c|c|}
\hline \multirow[t]{2}{*}{ Min } & \multicolumn{3}{|c|}{ a. Sibling call duration } & \multicolumn{3}{|c|}{ b. Own call duration } \\
\hline & $d f$ & $F$ & $P$ & $d f$ & $F$ & $P$ \\
\hline 0 & 1, 5572 & 170.8 & $<0.0001$ & - & - & - \\
\hline 1 & 1, 5572 & 60.6 & $<0.0001$ & $\mathbf{1}, 9787$ & $10,985.9$ & $<0.000$ \\
\hline 2 & 1, 5572 & 70.6 & $<0.0001$ & 1,9787 & 119.2 & $<0.000$ \\
\hline 3 & 1, 5572 & 17.1 & $<0.0001$ & 1,9787 & 23.0 & $<\mathbf{0 . 0 0 0}$ \\
\hline 4 & 1,5572 & 19.5 & $<0.0001$ & 1,9787 & 1.1 & 0.30 \\
\hline 5 & 1, 5572 & 13.9 & 0.0002 & 1,9787 & 0.4 & 0.54 \\
\hline 6 & 1,5572 & 4.6 & 0.03 & 1,9787 & 0.2 & 0.66 \\
\hline 7 & 1,5572 & 0.7 & 0.41 & $\mathbf{1}, \mathbf{9 7 8 7}$ & 5.2 & 0.02 \\
\hline 8 & 1,5572 & 0.0 & 0.86 & 1,9787 & 0.9 & 0.34 \\
\hline 9 & 1,5572 & 0.4 & 0.51 & 1,9787 & 4.0 & 0.05 \\
\hline 10 & 1,5572 & 0.5 & 0.48 & 1,9787 & 0.1 & 0.80 \\
\hline
\end{tabular}

Terms in bold are significant $(p<0.05)$

Nestling nested in sibling pair was set as random factor

The latency that singleton nestlings took before calling after a playback call was related to the interaction between competitors' motivation in the vocal exchange during the training playback and the duration of the memory window (term "Motivation $\times$ Duration" in Table 2c). Nestlings called significantly more rapidly (with shorter calling latency) after we broadcast calls of the "demotivated" compared to "motivated" individual, but only if the memory window following the training playback did not exceed $1 \mathrm{~min}$ (Fig. 4; after 1 min silence: $0.905 \pm 0.433$ (estimate \pm s.e.), $\mathrm{F}_{1,22}=4.37, P=0.048$; after $15 \mathrm{~min}$ silence: $-0.263 \pm 0.374, \mathrm{~F}_{1,29}=0.49, P=0.49$; after 30 min silence: $0.065 \pm 0.337, \mathrm{~F}_{1,26}=0.04, P=0.85$; same models, but separately for each memory window). This effect was not significantly related to the number of times the training playback was broadcast (as terms "Motivation $\times$ Duration $\times$ Number" and "Motivation $\times$ Duration" were not significant, Table 2c). However, if we analysed the effect of competitor motivation according to the number of training playbacks, the only significant discrimination after $1 \mathrm{~min}$ of silence window was found after the 3rd testing playback (same models performed after 1 min of silent window, on the 1st testing playback: estimate: $1.2 \pm 1.2, \mathrm{~F}_{1.5}=0.98, P=0.37 ; 2$ nd testing playback: $0.2 \pm 0.6, \mathrm{~F}_{1,6}=0.82, P=0.037$; 3rd testing playback: $\left.1.1 \pm 0.3, \mathrm{~F}_{1,9}=11.35, P=0.008\right)$.

\section{Discussion}

By analysing vocal exchange between pairs of barn owl siblings, we found that the duration of calls of focal 
Table 2 Vocal response of barn owl nestlings to competitors previously heard in a vocal exchange

\begin{tabular}{|c|c|c|c|c|c|c|c|c|c|}
\hline \multirow[b]{2}{*}{ Effect } & \multicolumn{3}{|c|}{ a. Number of calls } & \multicolumn{3}{|c|}{ b. Call duration (s) } & \multicolumn{3}{|c|}{ c. Calling latency (s) } \\
\hline & $d f$ & $\mathrm{~F}$ & $P$ & $d f$ & $\mathrm{~F}$ & $P$ & $d f$ & $\mathrm{~F}$ & $P$ \\
\hline $\begin{array}{l}\text { Motivation of competitors during training playbacks } \\
\text { ("Motivated" or "demotivated") }\end{array}$ & 1,314 & 0.41 & 0.52 & 1,143 & 0.51 & 0.48 & 1,134 & 0.72 & 0.40 \\
\hline $\begin{array}{l}\text { Duration of memory window after previous training playback } \\
(1,15 \text { or } 30 \mathrm{~min})\end{array}$ & 2,314 & 1.13 & 0.33 & 2,140 & 0.21 & 0.81 & 2,134 & 0.06 & 0.94 \\
\hline $\begin{array}{l}\text { Number of training playbacks heard } \\
(1,2 \text { or } 3)\end{array}$ & 2,318 & 6.83 & 0.001 & 2,143 & 6.51 & 0.002 & 2,134 & 0.78 & 0.46 \\
\hline Order of competitor during testing playback (1st or 2 nd) & 1,314 & 2.55 & 0.14 & 1,140 & 0.28 & 0.60 & 1,134 & 0.28 & 0.60 \\
\hline Motivation $\times$ duration & 2,306 & 0.65 & 0.52 & 2,134 & 0.24 & 0.78 & 2,134 & 3.70 & 0.027 \\
\hline Motivation $\times$ number & 2,306 & 0.63 & 0.53 & 2,143 & 4.31 & 0.015 & 2,134 & 0.82 & 0.44 \\
\hline Duration $\times$ number & 4,306 & 1.48 & 0.20 & 4,134 & 1.02 & 0.40 & 4,134 & 0.14 & 0.96 \\
\hline Motivation $\times$ duration $\times$ number & 4,302 & 1.33 & 0.26 & 4,130 & 0.57 & 0.69 & 4,130 & 1.02 & 0.40 \\
\hline
\end{tabular}

Terms eliminated from initial models are italicized. Interactions in bold are described in the "Results" section

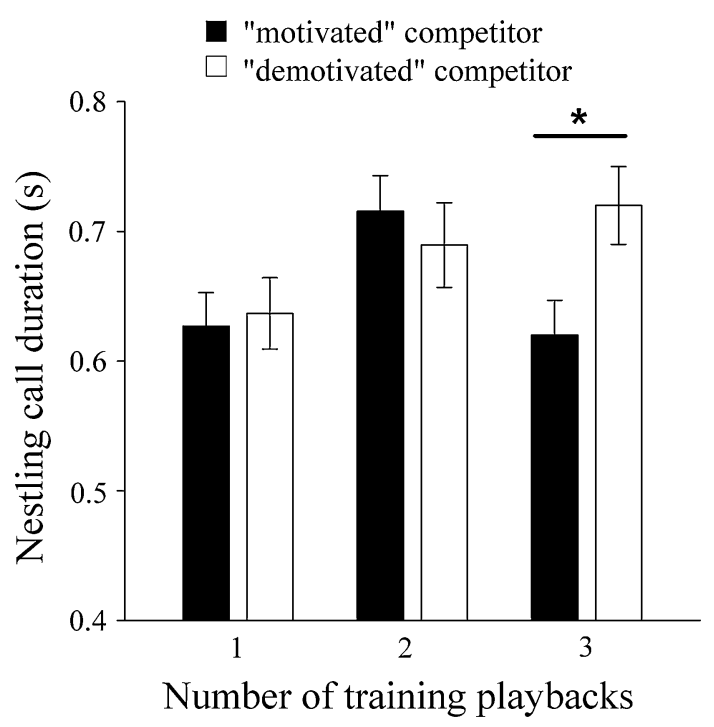

Fig. 3 Nestling call duration according to the previous motivation of competitors, witnessed once, twice or three times. Mean call duration (s) of singleton barn owl nestlings ( \pm s.e.) during testing playbacks, according to the number of training playbacks that these nestlings heard. Nestlings responded to competitors which formerly displayed a different level of motivation (during training playback). The broadcast individuals displayed either a high or low motivation with "motivated" individuals emitting 12 calls per minute and "demotivated" individuals 4 calls. The asterisk indicates significant difference in the mixed model reported in the results

nestlings was correlated with the duration of sibling's calls emitted up to 6 min before. This suggests that at each time point nestlings adjust their calling behaviour in relation to the way their siblings called up to 6 min before. Our playback experiment allowed us to test whether nestlings

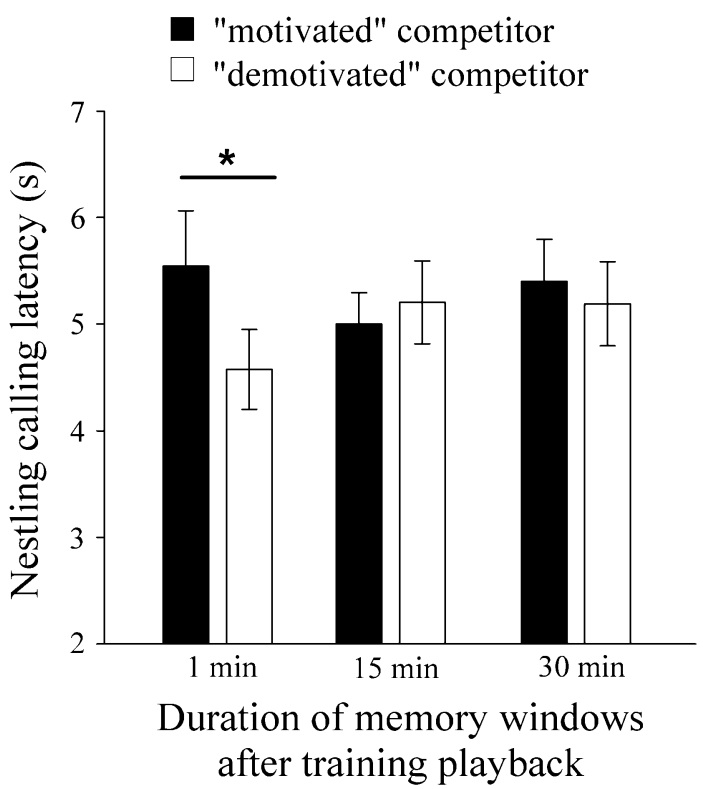

Fig. 4 Nestling calling latency according to the motivation of competitors witnessed 1,15 or $30 \mathrm{~min}$ before. Calling latency (s) of barn owl nestlings ( \pm s.e.), responding to competitors which formerly displayed a different level of motivation, according to the duration of silent memory window after the previous training playback. The calling latency was estimated as the mean amount of time between the beginning of a broadcast call and the beginning of the singleton nestling's call during testing playback. The asterisk indicates significant difference in the linear mixed model

identify which of two siblings is the hungriest and retain this information for longer periods of time to adjust their calling behaviour. We report the first test and evidence that young animals that are still dependent on their parents assess and memorize during at least 15 min the outcome of 
social interactions taking place between two opponents. Although the duration of memory reported here is not necessarily exceptional, the study question of whether memory plays a role in family interactions is not trivial. Indeed, existing theoretical models about sibling competition (Godfray 1995; Godfray and Parker 1992; Royle et al. 2012) implicitly consider that young animals assess current sibling's physical and signalling behaviour to adjust their own levels of effort invested in the competition over parental resources. In other words, the decision to escalate begging behaviour in a young animal is taken at the exact time when its siblings increase effort in sibling competition rather than based on previously witnessed siblings' behaviour (Leonard and Horn 1998; Madden et al. 2009). Furthermore, considering information retention has important implication on the honesty of signalling as discussed below.

\section{Information Retention During Natural Exchange}

To examine the extent to which nestlings memorize the calling behaviour of siblings to adjust their own calls, we made use of the observation that the duration of calls emitted by two barn owl siblings are correlated through time. In other words, when a nestling increases (or decreases) the duration of its calls, its sibling increases (vs. decreases) the duration of its own calls. Here, we therefore investigated whether the duration of calls produced by an individual is correlated with the duration of sibling's calls emitted several minutes ago. Our correlative analysis suggests that nestlings retained information on sibling calling behaviour for only a few minutes. During continuous vocal interaction between two nestlings, calls emitted by a sibling more than 6 min ago were no more taken into account by a focal nestling to adjust its vocal behaviour. This does not necessary mean that information cannot be memorized for a longer period of time, but that this information is constantly updated. Because we used a correlative approach, the adjustment of call duration could be related to other factors than own and sibling call duration that we did not control for. However, nestling modulation of call duration was unlikely due to hunger or circadian rhythm, as duration increases and decreases within a few minutes (Roulin et al. 2009). Furthermore, in our experiment siblings were physically separated, which limited other influences than acoustic signals.

Information Retention and Consolidation of Eavesdropped Vocalizations

Singleton nestlings modulate their investment in call duration according to the eavesdropped information even after $15 \mathrm{~min}$ of silence, but only after three training playbacks of 10 min, i.e. only if they could eavesdrop on competitors during a sufficiently long period of time. This finding is in line with the hypothesis that repetition of an eavesdropped vocal interaction consolidates individual ability to retain social information. The fact that significant discrimination was only found after $15 \mathrm{~min}$ of silence window might in part be due to a lack of statistical power as slightly fewer nestlings were calling during testing playback after 1 and $30 \mathrm{~min}$ (see degrees of freedom).

In line with the hypothesis that nestlings' ability to remember vocal behaviour of competitors decreased with time, nestlings also adjusted the time taken to call after a competitor (i.e. calling latency), but only if they witnessed the motivation of competitors no more than $1 \mathrm{~min}$ ago. This latter result confirms that nestlings adjust their calling latency to recently eavesdropped information (Dreiss et al. 2013a). Our results hence suggest that the vocal signals of competitors' motivation must be recent or witnessed $30 \mathrm{~min}$ to be remembered or taken into account. Four alternative scenarios could explain why nestlings adjust their call duration to former competitors' motivation only if witnessed during a sufficiently long period of time (i.e. after three training playbacks in our experimental setup). First, nestlings may have been incapable to discriminate between the two opponents after the first and second training playbacks of $10 \mathrm{~min}$, because they did not have time to identify which individual was calling at high level. Indeed, in our previous eavesdrop experiment (Dreiss et al. 2013a) that induced differential vocal responses in singleton nestlings to the two broadcast nestlings, the training playback was much longer (26 min). Second, this lack of discrimination response after the first and second training could be attributed to a lack of memory (no recollection of former interaction after silence window) or, third, to a decision to ignore the collected information. Forgetting or not taking into account information may be adaptive in barn owl nestlings if information is no more valid or if this constrains sibling competitors to repeat their display of motivation (see discussion below). Fourth, as more than $1 \mathrm{~h}$ has elapsed between the first and the third testing playbacks, the increased discrimination between "motivated" and "demotivated" could be related to the slightly increased hunger level of experimental nestlings. This seems however unlikely, as an individual is expected to vocalize relatively less intensely when facing "motivated" than "demotivated" nestmates independently of its own level of hunger.

Implication of Information Retention on the Honesty of Signalling

Information retention is predicted to be long when (1) the gathered information remains valid during a sufficiently 
long period of time, (2) memory is not too costly and (3) the benefit of memory retention is high. Information retention is expected to be shorter in variable than in stable environments, as information retained is less likely to persist over a long period of time, implying that signals have to be updated to convey the correct information (McNamara and Houston 1987). For instance, Rana sylvatica tadpoles remember the odour of a predator after a longer period of time when odour was associated with a high rather than low threat (Ferrari et al. 2010) and with a high rather than low certainty of threat (Ferrari et al. 2012). The ability to memorize social information is expected to evolve and be maintained if memory retention is not too costly. In very large groups, investment in memory of all group members may be cognitively costly, a situation that may select for direct assessment rather than memory of past social interactions to optimize behaviour when competing with a given individual. For instance, gelada Theropithecus gelada do not recognize adult males they had met outside their small reproductive group (le Roux and Bergman 2012). Even if primates are known for their high social cognition ability, living in large social groups $(>1,000$ individuals in gelada) may increase the cost/benefit ratio of memory (le Roux and Bergman 2012; Bergman 2010). In the barn owl, the benefit of remembering the displayed motivation level of competitor siblings is based on the verified assumption that owlets reduce investment in vocalization behaviour when facing a highly competitive and hence vocal sibling (Johnstone and Roulin 2003). Because the probability of winning the contest over a prey item delivered by a parent is low in front of hungry siblings, nestlings would spare energy by avoiding to challenge hungry siblings that are motivated to compete and hence likely to monopolize the impending food resources (Johnstone and Roulin 2003). We show here that nestlings indeed remember the level of motivation displayed at least 15 min ago. Nestling memory ability can hence allow individuals to spread signal investment over time. Hungry individuals can indeed stop calling for a while and still elicit a withdrawal from their nestmates who remember the level of motivation, reducing the global cost of sibling competition.

However, barn owl nestlings' memory of vocal competition is expected to be short for several reasons. First, the hunger levels of siblings change continuously during the course of the night (but probably not over $15 \mathrm{~min}$ unless an individual has been fed) and along the feeding events (Roulin 2002b). Because of the dynamic nature of sibling competition, information on competitors' motivation should lose value and become obsolete relatively rapidly. Second, in a competitive context, we speculate that the absence of memory would oblige conspecifics to repeat a display on several occasions, which would reinforce the honesty of communication. If signalling is energetically costly to produce, repetition could enhance the accuracy of the estimate of conspecific motivation (Payne and Pagel 1997, 1996). By taking into account only the signals emitted by competitors that repeatedly advertise their motivation level and only when the vocal signal has been emitted less than a few minutes ago may be a way to ensure the honesty of this vocal signal of hunger. Hence, to ensure that the emitted vocal signals of need are taken into account by siblings, nestlings would have to constantly repeat their display until parents come back at the nest with food. Accordingly, nestlings are producing thousands of calls during a single night (Roulin 2002a).

\section{Differential Use of Information Retention on Different Vocal Features}

Barn owl nestlings adjusted two out of three call features to the motivation of competitors witnessed in a previous training playback: call duration and calling latency, but not the number of calls. Call rate is more closely related to hunger level and more sensitive to current vocal competition than call duration (Ruppli et al. 2013a). Furthermore, compared to the duration of calls, the number of calls produced in parent absence is a better predictor of which nestling will monopolize the next delivered food item brought by a parent (Dreiss et al. 2010b). Since call rate is more related to the time spent vocalizing (and hence to the amount of vocal sound produced) than call duration and calling latency, call rate may be more costly to produce than long calls (Ruppli et al. 2013a) and calling rapidly after a sibling. Although the three different vocal features all signal motivation to compete over parental food resources (Dreiss et al. 2013a; Roulin et al. 2009), they play a slightly different role in the vocal contest. Interestingly, it appears that only less costly vocal features, i.e. call duration and calling latency, are modulated according to past motivation of competitors. In contrast, the more reliable component of vocal signals, i.e. call rate, is adjusted to the current level of siblings' signals which is probably more closely associated to the exact level of sibling motivation.

\section{Memory of Siblings' Identity}

The finding that barn owl nestlings responded differently to the two broadcast nestlings during the testing playback implies that they remembered the identity and past motivation level of competitor siblings experienced during the training playback. This ability can be explained by the fact that nestlings learnt the vocal signature (Dreiss et al. 2014) of the two broadcast nestlings and simultaneously measured their relative or absolute motivation level. 
Alternatively, they could have learnt the identity of only one of the two broadcast individuals (e.g. the "motivated" but not "demotivated" one) and in turn responded differently to this competitor compared to the forgotten individual. The later hypothesis seems unlikely because a previous study showed that nestlings assess the relative age rather than absolute age between two vocally competing siblings and the relative timing of their calls (Dreiss et al. 2013a). This finding suggests that they compare the vocal behaviours of the two broadcast individuals and rank them.

In our experiment, the difference in motivation to compete between the two broadcast competitors during the vocal exchange was not very pronounced (the "motivated" individual called only at a three times higher rate than the "demotivated" individual). It would be interesting to further test whether the intensity of the signal (in terms of call duration and call rate) affects the amount of time the information is taken into account, in the same way as the duration of the signal (in terms of number of training playbacks).

\section{Conclusion}

Behavioural adjustment in sibling competition may not necessarily require memory of previous sib-sib interactions, but sometimes solely depends on individual own need and on the intensity with which their surrounding siblings are currently contesting the same pool of parental resources (Madden et al. 2009; Godfray 1995; Leonard and Horn 1998, 2001). However, repeated interactions have long-lasting consequences on the establishment of a hierarchy among the siblings in some species (Drummond 2006) or on nestling signalling behaviour (Kedar et al. 2000; Grodzinski et al. 2008). The present study, as well as the previous study on the ability to eavesdrop on siblings competitive interactions (Dreiss et al. 2013a) showed that in barn owl nestlings the investment in sibling competition could be understood in the light of past vocal behaviour. Modulation of barn owl behaviour in relation to the competitive environment is hence a dynamic process that depends on both past (Dreiss et al. 2013a) and current interactions (Roulin et al. 2009). Our study underlines the importance of considering the dynamics of iterative interaction to understand the resolution of animal conflicts.

\section{References}

Aquiloni, L., \& Gherardi, F. (2010). Crayfish females eavesdrop on fighting males and use smell and sight to recognize the identity of the winner. Animal Behaviour, 79(2), 265-269.
Bergman, T. J. (2010). Experimental evidence for limited vocal recognition in a wild primate: Implications for the social complexity hypothesis. Proceedings of the Royal Society B, 277(1696), 3045-3053. doi:10.1098/rspb.2010.0580.

Boeckle, M., \& Bugnyar, T. (2012). Long-term memory for affiliates in ravens. Current Biology, 22(9), 801-806. doi:10.1016/j.cub. 2012.03.023.

Brown, G. E., Rive, A. C., Ferrari, M. C. O., \& Chivers, D. P. (2006). The dynamic nature of antipredator behavior: Prey fish integrate threat-sensitive antipredator responses within background levels of predation risk. Behavioral Ecology and Sociobiology, 61(1), 9-16. doi:10.1007/s00265-006-0232-y.

Castellano, S., Cadeddu, G., \& Cermelli, P. (2012). Computational mate choice: Theory and empirical evidence. Behavioural Processes, 90(2), 261-277. doi:10.1016/j.beproc.2012.02.010.

Clayton, N. S., \& Dickinson, A. (1998). Episodic-like memory during cache recovery by scrub jays. Nature, 395(6699), 272-274. doi: $10.1038 / 26216$.

Dedeo, S., Krakauer, D. C., \& Flack, J. C. (2010). Inductive game theory and the dynamics of animal conflict. PLoS Computational Biology, 6(5), e1000782. doi:10.1371/journal.pcbi.1000782.

Devenport, L., Hill, T., Wilson, M., \& Ogden, E. (1997). Tracking and averaging in variable environments: A transition rule. Journal of Experimental Psychology-Animal Behavior Processes, 23(4), 450-460. doi:10.1037//0097-7403.23.4.450.

Dreiss, A. N., Henry, I., Ruppli, C., Almasi, B., \& Roulin, A. (2010a). Darker eumelanic barn owls better withstand food depletion through resistance to food deprivation and lower appetite. Oecologia, 164(1), 65-71.

Dreiss, A. N., Lahlah, N., \& Roulin, A. (2010b). How siblings adjust sib-sib communication and begging signals to each other. Animal Behaviour, 80, 1049-1055.

Dreiss, A. N., Ruppli, C. A., Faller, C., \& Roulin, A. (2013a). Big brother is watching you: eavesdropping to resolve family conflicts. Behavioral Ecology, 24(3), 717-722. doi:10.1093/ beheco/ars 210.

Dreiss, A. N., Ruppli, C. A., Oberli, F., Antoniazza, S., Henry, I., \& Roulin, A. (2013b). Barn owls do not interrupt their siblings. Animal Behaviour, 86, 119-126. doi:10.1016/j.anbehav.2013.04. 019.

Dreiss, A. N., Ruppli, C. A., \& Roulin, A. (2014). Individual vocal signatures in barn owl nestlings: Does individual recognition have an adaptive role in sibling vocal competition? Journal of Evolutionary Biology, 27(1), 63-75. doi:10.1111/jeb.1227.

Drummond, H. (2006). Dominance in vertebrate broods and litters. Quarterly Review of Biology, 81(1), 3-32.

Dukas, R. (1999). Costs of memory: Ideas and predictions. Journal of Theoretical Biology, 197(1), 41-50. doi:10.1006/jtbi.1998.0856.

Dunlap, A. S., McLinn, C. M., MacCormick, H. A., Scott, M. E., \& Kerr, B. (2009). Why some memories do not last a lifetime: Dynamic long-term retrieval in changing environments. Behavioral Ecology, 20(5), 1096-1105. doi:10.1093/beheco/arp102.

Durant, J. M., \& Handrich, Y. (1998). Growth and food requirement flexibility in captive chicks of the European barn owl (Tyto alba). Journal of Zoology, 245(2), 137-145.

Erne, N., \& Amrhein, V. (2008). Long-term influence of simulated territorial intrusions on dawn and dusk singing in the Winter Wren: Spring versus autumn. Journal of Ornithology, 149(4), 479-486. doi:10.1007/s10336-008-0288-8.

Ferrari, M. C. O., Brown, G. E., Bortolotti, G. R., \& Chivers, D. P. (2010). Linking predator risk and uncertainty to adaptive forgetting: A theoretical framework and empirical test using tadpoles. Proceedings of the Royal Society B-Biological Sciences, 277(1691), 2205-2210. doi:10.1098/rspb.2009.2117.

Ferrari, M. C. O., Vrtelova, J., Brown, G. E., \& Chivers, D. P. (2012). Understanding the role of uncertainty on learning and retention 
of predator information. Animal Cognition, 15(5), 807-813. doi:10.1007/s10071-012-0505-y.

Gherardi, F., \& Atema, J. (2005). Memory of social partners in hermit crab dominance. Ethology, 111(3), 271-285.

Godard, R. (1991). Long-term memory of individual neighbours in a migratory songbird. Nature, 350, 228-229.

Godfray, H. C. J. (1995). Signaling of need between parents and young-parent-offspring conflict and sibling rivalry. American Naturalist, 146(1), 1-24.

Godfray, H. C. J., \& Parker, G. A. (1992). Clutch size, fecundity and parent-offspring conflict. Philosophical Transactions of the Royal Society B-Biological Sciences, 332, 67-79.

Greggers, U., \& Menzel, R. (1993). Memory dynamics and foraging strategies of honeybees. Behavioral Ecology and Sociobiology, 32(1), 17-29.

Grodzinski, U., Erev, I., \& Lotem, A. (2008). Can hungry nestlings be trained to reduce their begging? Behavioral Ecology, 19(1), 116-125. doi:10.1093/beheco/arm107.

Grosenick, L., Clement, T. S., \& Fernald, R. D. (2007). Fish can infer social rank by observation alone. Nature, 445(7126), 429-432. doi:10.1038/nature 05511 .

Joe, H., \& Zhu, R. (2005). Generalized Poisson distribution: The property of mixture of Poisson and comparison with negative binomial distribution. Biometrical Journal, 47(2), 219-229. doi:10.1002/bimj.200410102.

Johnstone, R. A., \& Roulin, A. (2003). Sibling negotiation. Behavioral Ecology, 14(6), 780-786.

Kedar, H., Rodriguez-Girones, M. A., Yedvab, S., Winkler, D. W., \& Lotem, A. (2000). Experimental evidence for offspring learning in parent-offspring communication. Proceedings of the Royal Society B-Biological Sciences, 267(1454), 1723-1727.

Kendrick, K. M., da Costa, A. P., Leigh, A. E., Hinton, M. R., \& Peirce, J. W. (2001). Sheep don't forget a face. Nature, 414(6860), 165-166. doi:10.1038/35102669.

Killeen, P. R. (1981). Averaging theory. In M. C. Bradshaw, E. Szabadi, \& C. F. Lowe (Eds.), Quantification of steady state operant behavior (pp. 21-34). Amsterdam: Elsevier.

le Roux, A., \& Bergman, T. J. (2012). Indirect rival assessment in a social primate, Theropithecus gelada. Animal Behaviour, 83, 249-255.

Leonard, M. L., \& Horn, A. G. (1998). Need and nestmates affect begging in tree swallows. Behavioral Ecology and Sociobiology, 42(6), 431-436.

Leonard, M. L., \& Horn, A. G. (2001). Dynamics of calling by tree swallow (Tachycineta bicolor) nestmates. Behavioral Ecology and Sociobiology, 50(5), 430-435.

Madden, J. R., Kunc, H. P., English, S., Manser, M. B., \& CluttonBrock, T. H. (2009). Calling in the gap: Competition or cooperation in littermates' begging behaviour? Proceedings of the Royal Society B-Biological Sciences, 276(1660), 1255-1262.

Martin, T. E. (1995). Avian life history evolution in relation to nest sites, nest predation and food. Ecological Monographs, 65, 101-127.
McGregor, P. K. (1993). Signaling in territorial systems-A context for individual identification, ranging and eavesdropping. Philosophical Transactions of the Royal Society of London Series B-Biological Sciences, 340(1292), 237-244.

McNamara, J. M., \& Houston, A. I. (1987). Memory and the efficient use of information. Journal of Theoretical Biology, 125(4), 385-395. doi:10.1016/s0022-5193(87)80209-6.

Mennill, D. J., \& Ratcliffe, L. M. (2004). Do male black-capped chickadees eavesdrop on song contests? A multi-speaker playback experiment. Behaviour, 141, 125-139.

Mery, F., \& Kawecki, T. J. (2005). A cost of long-term memory in Drosophila. Science, 308(5725), 1148. doi:10.1126/science. 111131.

Moreira, J., Vukov, J., Sousa, C., Santos, F. C., d'Almeida, A. F., Santos, M. D., et al. (2013). Individual memory and the emergence of cooperation. Animal Behaviour, 85(1), 233-239. doi:10.1016/j.anbehav.2012.10.030.

Payne, R. J. H., \& Pagel, M. (1996). Escalation and time costs in displays of endurance. Journal of Theoretical Biology, 183(2), 185-193. doi:10.1006/jtbi.1996.0212.

Payne, R. J. H., \& Pagel, M. (1997). Why do animals repeat displays? Animal Behaviour, 54, 109-119. doi:10.1006/anbe.1996.0391.

Roulin, A. (2002a). The sibling negotiation hypothesis. In J. Wright \& M. L. Leonard (Eds.), The evolution of begging: Competition, cooperation and communication (pp. 107-127). Dordrecht: Kluwer Academic Press.

Roulin, A. (2002b). Tyto alba Barn Owl. BWP Update, 4, 115-138.

Roulin, A. (2004). The function of food stores in bird nests: observations and experiments in the barn owl Tyto alba. Ardea, 92(1), 69-78.

Roulin, A., \& Bersier, L. F. (2007). Nestling barn owls beg more intensely in the presence of their mother than in the presence of their father. Animal Behaviour, 74, 1099-1106.

Roulin, A., Dreiss, A. N., Fioravanti, C., \& Bize, P. (2009). Vocal sib-sib interactions: How siblings adjust signalling level to each other. Animal Behaviour, 77(3), 717-725.

Roulin, A., Kolliker, M., \& Richner, H. (2000). Barn owl (Tyto alba) siblings vocally negotiate resources. Proceedings of the Royal Society B-Biological Sciences, 267(1442), 459-463.

Royle, N. J., Smiseth, P. T., \& Kölliker, M. (2012). The evolution of parental care. Oxford: Oxford University Press.

Ruppli, C. A., Dreiss, A. N., \& Roulin, A. (2013a). Efficiency and significance of multiple vocal signals in sibling competition. Evolutionary Biology, 40(4), 579-588. doi:10.1007/s11692-0139233-8.

Ruppli, C. A., Dreiss, A. N., \& Roulin, A. (2013b). Nestling barn owls assess short-term variation in the amount of vocally competing siblings. Animal Cognition, 16(6), 993-1000. doi:10.1007/ s10071-013-0634-y.

Schmidt, R., Amrhein, V., Kunc, H. P., \& Naguib, M. (2007). The day after: Effects of vocal interactions on territory defence in nightingales. Journal of Animal Ecology, 76(1), 168-173. doi:10. 1111/j.1365-2656.2006.01182.x. 